\title{
On Paranorm Zweier I-Convergent Sequence Spaces
}

\author{
Vakeel A. Khan, ${ }^{1}$ Khalid Ebadullah, ${ }^{1}$ Ayhan Esi, ${ }^{2}$ Nazneen Khan, ${ }^{1}$ and Mohd Shafiq ${ }^{1}$ \\ ${ }^{1}$ Department of Mathematics, Aligarh Muslim University, Aligarh 202002, India \\ ${ }^{2}$ Department of Mathematics, University of Adiyaman, Altinsehir, 02040 Adiyaman, Turkey
}

Correspondence should be addressed to Vakeel A. Khan; vakhan@math.com

Received 14 August 2012; Revised 6 November 2012; Accepted 21 November 2012

Academic Editor: Ali Jaballah

Copyright (C) 2013 Vakeel A. Khan et al. This is an open access article distributed under the Creative Commons Attribution License, which permits unrestricted use, distribution, and reproduction in any medium, provided the original work is properly cited.

In this paper, we introduce the paranorm Zweier $I$-convergent sequence spaces $\mathscr{Z}^{I}(q), \mathscr{Z}_{I}^{0}(q)$, and $\mathscr{Z}_{\infty}^{I}(q) \mathscr{Z}_{\infty}^{I}(q)$ for $q=\left(q_{k}\right)$, a sequence of positive real numbers. We study some topological properties, prove the decomposition theorem, and study some inclusion relations on these spaces.

\section{Introduction}

Let $\mathbb{N}, \mathbb{R}$, and $\mathbb{C}$ be the sets of all natural, real, and complex numbers, respectively. We write

$$
\omega=\left\{x=\left(x_{k}\right): x_{k} \in \mathbb{R} \text { or } \mathbb{C}\right\}
$$

the space of all real or complex sequences.

Let $l_{\infty}, c$, and $c_{0}$ denote the Banach spaces of bounded, convergent, and null sequences, respectively, normed by $\|x\|_{\infty}=\sup _{k}\left|x_{k}\right|$.

The following subspaces of $\omega$ were first introduced and discussed by Maddox [1]:

$$
\begin{aligned}
& l(p):=\left\{x \in \omega: \sum_{k}\left|x_{k}\right|^{p_{k}}<\infty\right\}, \\
& l_{\infty}(p):=\left\{x \in \omega: \sup _{k}\left|x_{k}\right|^{p_{k}}<\infty\right\}, \\
& c(p):=\left\{x \in \omega: \lim _{k}\left|x_{k}-l\right|^{p_{k}}=0, \text { for some } l \in \mathbb{C}\right\}, \\
& c_{0}(p):=\left\{x \in \omega: \lim _{k}\left|x_{k}\right|^{p_{k}}=0\right\},
\end{aligned}
$$

where $p=\left(p_{k}\right)$ is a sequence of strictly positive real numbers.
After that Lascarides [2,3] defined the following sequence spaces:

$$
\begin{array}{r}
l_{\infty}\{p\}=\{x \in \omega: \text { there exists } r>0 \\
\text { such that } \left.\sup _{k}\left|x_{k} r\right|^{p_{k}} t_{k}<\infty\right\}, \\
c_{0}\{p\}=\{x \in \omega: \text { there exists } r>0 \\
\text { such that } \left.\lim _{k}\left|x_{k} r\right|^{p_{k}} t_{k}=0\right\}, \\
l\{p\}=\{x \in \omega: \text { there exists } r>0 \\
\text { such that } \left.\sum_{k=1}^{\infty}\left|x_{k} r\right|^{p_{k}} t_{k}<\infty\right\},
\end{array}
$$

where $t_{k}=p_{k}^{-1}$, for all $k \in \mathbb{N}$.

Each linear subspace of $\omega$, for example, $\lambda, \mu \subset \omega$, is called a sequence space.

A sequence space $\lambda$ with linear topology is called a $K$ space provided each map $p_{i} \rightarrow \mathbb{C}$ defined by $p_{i}(x)=x_{i}$ is continuous for all $i \in \mathbb{N}$.

A $K$-space $\lambda$ is called an FK-space provided $\lambda$ is a complete linear metric space.

An FK-space whose topology is normable is called a BKspace.

Let $\lambda$ and $\mu$ be two sequence spaces and $A=\left(a_{n k}\right)$ an infinite matrix of real or complex numbers $a_{n k}$, where $n, k \in \mathbb{N}$. 
Then we say that $A$ defines a matrix mapping from $\lambda$ to $\mu$, and we denote it by writing $A: \lambda \rightarrow \mu$.

If for every sequence $x=\left(x_{k}\right) \in \lambda$ the sequence $A x=$ $\left\{(A x)_{n}\right\}$, the $A$ transform of $x$ is in $\mu$, where

$$
(A x)_{n}=\sum_{k} a_{n k} x_{k}, \quad(n \in \mathbb{N}) .
$$

By $(\lambda: \mu)$, we denote the class of matrices $A$ such that $A$ : $\lambda \rightarrow \mu$.

Thus, $A \in(\lambda: \mu)$ if and only if series on the right side of (3) converges for each $n \in \mathbb{N}$ and every $x \in \lambda$.

The approach of constructing the new sequence spaces by means of the matrix domain of a particular limitation method has been recently employed by Altay et al. [4], Başar and Altay [5], Malkowsky [6], Ng and Lee [7], and Wang [8].

Şengönül [9] defined the sequence $y=\left(y_{i}\right)$ which is frequently used as the $Z^{p}$ transform of the sequence $x=\left(x_{i}\right)$, that is,

$$
y_{i}=p x_{i}+(1-p) x_{i-1}
$$

where $x_{-1}=0, p \neq 1,1<p<\infty$ and $Z^{p}$ denotes the matrix $Z^{p}=\left(z_{i k}\right)$ defined by

$$
\mathscr{Z}_{i k}= \begin{cases}p, & (i=k), \\ 1-p, & (i-1=k) ;(i, k \in \mathbb{N}), \\ 0, & \text { otherwise. }\end{cases}
$$

Following Başar and Altay [5], Şengönül [9] introduced the Zweier sequence spaces $\mathscr{Z}$ and $\mathscr{Z}_{0}$ as follows:

$$
\begin{aligned}
\mathscr{Z} & =\left\{x=\left(x_{k}\right) \in \omega: Z^{p} x \in c\right\}, \\
\mathscr{Z}_{0} & =\left\{x=\left(x_{k}\right) \in \omega: Z^{p} x \in c_{0}\right\} .
\end{aligned}
$$

Here we quote below some of the results due to Şengönül [9] which we will need in order to establish the results of this paper.

Theorem 1 (see [9, Theorem 2.1]). The sets $\mathscr{Z}$ and $\mathscr{Z}_{0}$ are the linear spaces with the coordinate wise addition and scalar multiplication which are the BK-spaces with the norm

$$
\|x\|_{\mathscr{Z}}=\|x\|_{\mathscr{E}_{0}}=\left\|Z^{p} x\right\|_{c} .
$$

Theorem 2 (see [9, Theorem 2.2]). The sequence spaces $\mathscr{Z}$ and $\mathscr{Z}_{0}$ are linearly isomorphic to the spaces $c$ and $c_{0}$, respectively, that is, $\mathscr{Z} \cong c$ and $\mathscr{Z}_{0} \cong c_{0}$.

Theorem 3 (see [9, Theorem 2.3]). The inclusions $\mathscr{Z}_{0} \subset \mathscr{Z}$ strictly hold for $p \neq 1$.

Theorem 4 (see [9, Theorem 2.6]). $\mathscr{Z}_{0}$ is solid.

Theorem 5 (see [9, Theorem 3.6]). $\mathscr{Z}$ is not a solid sequence space.

The concept of statistical convergence was first introduced by Fast [10] and also independently by Buck [11] and
Schoenberg [12] for real and complex sequences. Further this concept was studied by Connor [13, 14], Connor et al. [15], and many others. Statistical convergence is a generalization of the usual notion of convergence that parallels the usual theory of convergence. A sequence $x=\left(x_{k}\right)$ is said to be statistically convergent to $L$ if for a given $\epsilon>0$ as

$$
\lim _{k} \frac{1}{k}\left|\left\{i:\left|x_{i}-L\right| \geq \epsilon, i \leq k\right\}\right|=0 .
$$

The notion of $I$-convergence is a generalization of the statistical convergence. At the initial stage, it was studied by Kostyrko et al. [16]. Later on, it was studied by Šalát et al. $[17,18]$, Demirci [19], Tripathy and Hazarika [20, 21], and Khan et al. [22-24].

Here we give some preliminaries about the notion of $I$ convergence.

Let $\mathrm{X}$ be a nonempty set. Then a family of sets $I \subseteq 2^{X}$ (denoting the power set of $X$ ) is said to be an ideal if $I$ is additive, that is, $A, B \in I \Rightarrow A \cup B \in I$, and hereditary, that is, $A \in I, B \subseteq A \Rightarrow B \in I$.

A nonempty family of sets $£ \subseteq 2^{X}$ is said to be a filter on $X$ if and only if $\phi \notin \mathfrak{E}$, for $A, B \in \mathfrak{E}$ we have $A \cap B \in \mathfrak{E}$ and for each $A \in \mathfrak{E}$ and $A \subseteq B$ implies $B \in \mathfrak{E}$.

An ideal $I \subseteq 2^{X}$ is called nontrivial if $I \neq 2^{X}$.

A non-trivial ideal $I \subseteq 2^{X}$ is called admissible if $\{\{x\}$ : $x \in X\} \subseteq I$.

A non-trivial ideal $I$ is maximal if there cannot exist any non-trivial ideal $J \neq I$ containing $I$ as a subset.

For each ideal $I$, there is a filter $\mathfrak{E}(I)$ corresponding to $I$. that is, $\mathfrak{E}(I)=\left\{K \subseteq N: K^{c} \in I\right\}$, where $K^{c}=N-K$.

Definition 6. A sequence $\left(x_{k}\right) \in \omega$ is said to be $I$-convergent to a number $L$ if $\left\{k \in N:\left|x_{k}-L\right| \geq \epsilon\right\} \in I$ for every $\varepsilon>0$. In this case we write $I-\lim x_{k}=L$.

The space $c^{I}$ of all $I$-convergent sequences converging to $L$ is given by

$$
\begin{gathered}
c^{I}=\left\{\left(x_{k}\right) \in \omega:\left\{k \in \mathbb{N}:\left|x_{k}-L\right| \geq \epsilon\right\} \in I,\right. \\
\text { for some } L \in \mathbb{C}\} .
\end{gathered}
$$

Definition 7. A sequence $\left(x_{k}\right) \in \omega$ is said to be $I$-null if $L=0$. In this case we write $I-\lim x_{k}=0$.

Definition 8. A sequence $\left(x_{k}\right) \in \omega$ is said to be $I$-Cauchy if for every $\epsilon>0$ there exists a number $k_{0} \in \mathbb{N}$ such that $\{k \epsilon$ $\left.N:\left|x_{k}-x_{m}\right| \geq \epsilon\right\} \in I$ for all $k, m \geq k_{0}$.

Definition 9. A sequence $\left(x_{k}\right) \in \omega$ is said to be $I$-bounded if there exists $M>0$ such that $\left\{k \in N:\left|x_{k}\right|>M\right\} \in I$.

Definition 10. Let $\left(x_{k}\right),\left(y_{k}\right)$ be two sequences. We say that $\left(x_{k}\right)=\left(y_{k}\right)$ for almost all $k$ relative to $I$ (a.a.k.r.I), if $\{k \in \mathbb{N}$ : $\left.x_{k} \neq y_{k}\right\} \in I$.

The following lemma will be used for establishing some results of this paper.

Lemma 11. If $I \subset 2^{N}$ and $M \subseteq N$. If $M \notin I$, then $M \cap N \notin I$ (see $[20,21]) c f .([17,18,20-24])$. 
Recently Khan and Ebadullah [25] introduced the following classes of sequence spaces:

$$
\begin{gathered}
\mathscr{Z}^{I}=\left\{k \in \mathbb{N}:\left\{x=\left(x_{k}\right) \in \omega: I-\lim Z^{p} x=L\right.\right. \\
\text { for some } L\}\} \in I, \\
\mathscr{Z}_{0}^{I}=\left\{k \in \mathbb{N}:\left\{x=\left(x_{k}\right) \in \omega: I-\lim Z^{p} x=0\right\}\right\} \in I, \\
\mathscr{Z}_{\infty}^{I}=\left\{k \in \mathbb{N}:\left\{x=\left(x_{k}\right) \in \omega: \sup _{k}\left|Z^{p} x\right|<\infty\right\}\right\} \in I,
\end{gathered}
$$

We also denote by

$$
m_{\mathscr{Z}}^{I}=\mathscr{Z}_{\infty}^{I} \cap \mathscr{Z}^{I}, \quad m_{\mathscr{E}_{0}}^{I}=\mathscr{E}_{\infty}^{I} \cap \mathscr{E}_{0}^{I} .
$$

In this paper we introduce the following classes of sequence spaces:

$$
\begin{aligned}
& \mathscr{Z}^{I}(q)=\left\{x=\left(x_{k}\right) \in \omega:\left\{k \in \mathbb{N}:\left|Z^{p} x-L\right|^{q_{k}} \geq \epsilon\right\} \in I,\right. \\
& \quad \text { for some } L \in \mathbb{C}\} ; \\
& \mathscr{Z}_{0}^{I}(q)=\left\{x=\left(x_{k}\right) \in \omega:\left\{k \in \mathbb{N}:\left|Z^{p} x\right|^{q_{k}} \geq \epsilon\right\} \in I\right\} ; \\
& \mathscr{I}_{\infty}^{I}(q)=\left\{x=\left(x_{k}\right) \in \omega: \sup _{k}\left|Z^{p} x\right|^{q_{k}}<\infty\right\} .
\end{aligned}
$$

We also denote by

$$
\begin{aligned}
& m_{\mathscr{Z}}^{I}(q)=\mathscr{Z}_{\infty}^{I}(q) \cap \mathscr{Z}^{I}(q), \\
& m_{\mathscr{E}_{0}}^{I}(q)=\mathscr{Z}_{\infty}^{I}(q) \cap \mathscr{I}_{0}^{I}(q),
\end{aligned}
$$

where $q=\left(q_{k}\right)$, is a sequence of positive real numbers.

Throughout the paper, for the sake of convenience now we will denote by $Z^{p} x=x^{\prime}, Z^{p} y=y^{\prime}, Z^{p} z=z^{\prime}$ for all $x, y, z \in \omega$.

\section{Main Results}

Theorem 12. The classes of sequences $\mathscr{Z}^{I}(q), \mathscr{Z}_{0}^{I}(q), m_{\mathscr{X}}^{I}(q)$ and $m_{\mathscr{E}_{0}}^{I}(q)$ are linear spaces.

Proof. We shall prove the result for the space $\mathscr{Z}^{I}(q)$.

The proof for the other spaces will follow similarly.

Let $\left(x_{k}\right),\left(y_{k}\right) \in \mathscr{Z}^{I}(q)$, and let $\alpha, \beta$ be scalars. Then for a given $\epsilon>0$ : we have

$$
\begin{aligned}
& \left\{k \in \mathbb{N}:\left|x_{k}^{\prime}-L_{1}\right|^{q_{k}} \geq \frac{\epsilon}{2 M_{1}}, \text { for some } L_{1} \in \mathbb{C}\right\} \in I \\
& \left\{k \in \mathbb{N}:\left|y_{k}^{\prime}-L_{2}\right|^{q_{k}} \geq \frac{\epsilon}{2 M_{2}}, \text { for some } L_{2} \in \mathbb{C}\right\} \in I,
\end{aligned}
$$

where

$$
\begin{aligned}
& M_{1}=D \cdot \max \left\{1, \underset{k}{\left.\sup |\alpha|^{q_{k}}\right\},}\right. \\
& M_{2}=D \cdot \max \left\{1, \sup _{k}|\beta|^{q_{k}}\right\}, \\
& D=\max \left\{1,2^{H-1}\right\} \quad \text { where } H=\sup _{k} q_{k} \geq 0 .
\end{aligned}
$$

Let

$$
\begin{aligned}
& A_{1}=\left\{k \in \mathbb{N}:\left|x_{k}^{l}-L_{1}\right|^{q_{k}}<\frac{\epsilon}{2 M_{1}}, \text { for some } L_{1} \in \mathbb{C}\right\} \in I \\
& A_{2}=\left\{k \in \mathbb{N}:\left|y_{k}^{l}-L_{2}\right|^{q_{k}}<\frac{\epsilon}{2 M_{2}}, \text { for some } L_{2} \in \mathbb{C}\right\} \in I
\end{aligned}
$$

be such that $A_{1}^{c}, A_{2}^{c} \in I$

Then

$$
\begin{aligned}
A_{3} & =\left\{k \in \mathbb{N}:\left|\left(\alpha x_{k}^{\prime}+\beta y_{k}^{\prime}\right)-\left(\alpha L_{1}+\beta L_{2}\right)\right|^{q_{k}}<\epsilon\right\} \\
& \supseteq\left\{k \in \mathbb{N}:|\alpha|^{q_{k}}\left|x_{k}^{\prime}-L_{1}\right|^{q_{k}}<\frac{\epsilon}{2 M_{1}}|\alpha|^{q_{k}} \cdot D\right\} \\
& \cap\left\{k \in \mathbb{N}:|\beta|^{q_{k}}\left|y_{k}^{\prime}-L_{2}\right|^{q_{k}}<\frac{\epsilon}{2 M_{2}}|\beta|^{q_{k}} \cdot D\right\} .
\end{aligned}
$$

Thus $A_{3}^{c}=A_{1}^{c} \cap A_{2}^{c} \in I$. Hence $\left(\alpha x_{k}+\beta y_{k}\right) \in \mathscr{Z}^{I}(q)$. Therefore $\mathscr{Z}^{I}(q)$ is a linear space. The rest of the result follows similarly.

Theorem 13. Let $\left(q_{k}\right) \in l_{\infty}$. Then $m_{\mathscr{E}}^{I}(q)$ and $m_{\mathscr{E}_{0}}^{I}(q)$ are paranormed spaces, paranormed by $g(x)=\sup _{k}\left|x_{k}\right| q_{k} / M$ where $M=\max \left\{1, \sup _{k} q_{k}\right\}$

Proof. Let $x=\left(x_{k}\right), y=\left(y_{k}\right) \in m_{\mathscr{I}}^{I}(q)$.

(1) Clearly, $g(x)=0$ if and only if $x=0$.

(2) $g(x)=g(-x)$ is obvious.

(3) Since $q_{k} / M \leq 1$ and $M>1$, using Minkowski's inequality, we have

$$
\sup _{k}\left|x_{k}+y_{k}\right|^{q_{k} / M} \leq \sup _{k}\left|x_{k}\right|^{q_{k} / M}+\sup _{k}\left|y_{k}\right|^{q_{k} / M}
$$

(4) Now for any complex $\lambda$, we have $\left(\lambda_{k}\right)$ such that $\lambda_{k} \rightarrow$ $\lambda,(k \rightarrow \infty)$.

Let $x_{k} \in m_{\mathscr{Z}}^{I}(q)$ such that $\left|x_{k}-L\right|^{q_{k}} \geq \epsilon$.

Therefore, $g(x-L e)=\sup _{k}\left|x_{k}-L\right|^{q_{k} / M} \leq \sup _{k}\left|x_{k}\right|^{q_{k} / M}+$ $\sup _{k}|L|^{q_{k} / M}$, where $e=(1,1,1, \ldots)$.

Hence $g\left(\left(\lambda_{n} x_{k}-\lambda L\right)\right) \leq g\left(\left(\lambda_{n} x_{k}\right)\right)+g(\lambda L)=\lambda_{n} g(x)+$ $\lambda g(L)$ as $(k \rightarrow \infty)$.

Hence $m_{\mathscr{E}}^{I}(q)$ is a paranormed space.

The rest of the result follows similarly. 
Theorem 14. $m_{\mathscr{E}}^{I}(q)$ is a closed subspace of $l_{\infty}(q)$.

Proof. Let $\left(x_{k}^{(n)}\right)$ be a Cauchy sequence in $m_{\mathscr{E}}^{I}(q)$ such that $x^{(n)} \rightarrow x$.

We show that $x \in m_{\mathscr{E}}^{I}(q)$.

Since $\left(x_{k}^{(n)}\right) \in m_{\mathscr{Z}}^{I}(q)$, then there exists $a_{n}$ such that

$$
\left\{k \in \mathbb{N}:\left|x^{(n)}-a_{n}\right| \geq \epsilon\right\} \in I .
$$

We need to show that

(1) $\left(a_{n}\right)$ converges to $a$,

(2) if $U=\left\{k \in \mathbb{N}:\left|x_{k}-a\right|<\epsilon\right\}$, then $U^{c} \in I$.

(1) Since $\left(x_{k}^{(n)}\right)$ is a Cauchy sequence in $m_{\mathscr{L}}^{I}(q)$ then for a given $\epsilon>0$, there exists $k_{0} \in \mathbb{N}$ such that

$$
\sup _{k}\left|x_{k}^{(n)}-x_{k}^{(i)}\right|<\frac{\epsilon}{3}, \quad \forall n, i \geq k_{0} .
$$

For a given $\varepsilon>0$, we have

$$
\begin{aligned}
& B_{n i}=\left\{k \in \mathbb{N}:\left|x_{k}^{(n)}-x_{k}^{(i)}\right|<\frac{\epsilon}{3}\right\}, \\
& B_{i}=\left\{k \in \mathbb{N}:\left|x_{k}^{(i)}-a_{i}\right|<\frac{\epsilon}{3}\right\}, \\
& B_{n}=\left\{k \in \mathbb{N}:\left|x_{k}^{(n)}-a_{n}\right|<\frac{\epsilon}{3}\right\} .
\end{aligned}
$$

Then $B_{n i}^{c}, B_{i}^{c}, B_{n}^{c} \in I$

Let $B^{c}=B_{n i}^{c} \cap B_{i}^{c} \cap B_{n}^{c}$, where $B=\left\{k \in \mathbb{N}:\left|a_{i}-a_{n}\right|<x \epsilon\right\}$.

Then $B^{c} \in I$.

We choose $k_{0} \in B^{c}$, then for each $n, i \geq k_{0}$, we have

$$
\begin{aligned}
\left\{k \in \mathbb{N}:\left|a_{i}-a_{n}\right|<\epsilon\right\} & \supseteq\left\{k \in \mathbb{N}:\left|x_{k}^{(i)}-a_{i}\right|<\frac{\epsilon}{3}\right\} \\
& \cap\left\{k \in \mathbb{N}:\left|x_{k}^{(n)}-x_{k}^{(i)}\right|<\frac{\epsilon}{3}\right\} \\
& \cap\left\{k \in \mathbb{N}:\left|x_{k}^{(n)}-a_{n}\right|<\frac{\epsilon}{3}\right\} .
\end{aligned}
$$

Then $\left(a_{n}\right)$ is a Cauchy sequence of scalars in $\mathbb{C}$, so there exists a scalar $a \in \mathbb{C}$ such that $a_{n} \rightarrow a$, as $n \rightarrow \infty$.

(2) Let $0<\delta<1$ be given. Then we show that if $U=\{k \in$ $\left.\mathbb{N}:\left|x_{k}-a\right|^{q_{k}}<\delta\right\}$, then $U^{c} \in I$.

Since $x^{(n)} \rightarrow x$, then there exists $q_{0} \in \mathbb{N}$ such that

$$
P=\left\{k \in \mathbb{N}:\left|x^{\left(q_{0}\right)}-x\right|<\left(\frac{\delta}{3 D}\right)^{M}\right\}
$$

which implies that $P^{c} \in I$.

The number $q_{0}$ can be so chosen that together with (23), we have

$$
Q=\left\{k \in \mathbb{N}:\left|a_{q_{0}}-a\right|^{q_{k}}<\left(\frac{\delta}{3 D}\right)^{M}\right\}
$$

such that $Q^{c} \in I$.
Since $\left\{k \in \mathbb{N}:\left|x^{\left(q_{0}\right)}-a_{q_{0}}\right|^{q_{k}} \geq \delta\right\} \in I$. Then we have a subset $S$ of $\mathbb{N}$ such that $S^{\mathcal{C}} \in I$, where

$$
S=\left\{k \in \mathbb{N}:\left|x^{\left(q_{0}\right)}-a_{q_{0}}\right|^{q_{k}}<\left(\frac{\delta}{3 D}\right)^{M}\right\} .
$$

Let $U^{c}=P^{c} \cap Q^{c} \cap S^{c}$, where $U=\left\{k \in \mathbb{N}:\left|x_{k}-a\right|^{q_{k}}<\delta\right\}$.

Therefore for each $k \in U^{c}$, we have

$$
\begin{aligned}
\{k & \left.\in \mathbb{N}:\left|x_{k}-a\right|^{q_{k}}<\delta\right\} \\
& \supseteq\left\{k \in \mathbb{N}:\left|x^{\left(q_{0}\right)}-x\right|^{q_{k}}<\left(\frac{\delta}{3 D}\right)^{M}\right\} \\
& \cap\left\{k \in \mathbb{N}:\left|x^{\left(q_{0}\right)}-a_{q_{0}}\right|^{q_{k}}<\left(\frac{\delta}{3 D}\right)^{M}\right\} \\
& \cap\left\{k \in \mathbb{N}:\left|a_{q_{0}}-a\right|^{q_{k}}<\left(\frac{\delta}{3 D}\right)^{M}\right\} .
\end{aligned}
$$

Then the result follows.

Since the inclusions $m_{\mathscr{X}}^{I}(q) \subset l_{\infty}(q)$ and $m_{\mathscr{E}_{0}}^{I}(q) \subset l_{\infty}(q)$ are strict, so in view of Theorem 14 we have the following result.

Theorem 15. The spaces $m_{\mathscr{E}}^{I}(q)$ and $m_{\mathscr{E}_{0}}^{I}(q)$ are nowhere dense subsets of $l_{\infty}(q)$.

Theorem 16. The spaces $m_{\mathscr{E}}^{I}(q)$ and $m_{\mathscr{E}_{0}}^{I}(q)$ are not separable.

Proof. We shall prove the result for the space $m_{\mathscr{E}}^{I}(q)$.

The proof for the other spaces will follow similarly.

Let $M$ be an infinite subset of $\mathbb{N}$ of increasing natural numbers such that $M \in I$.

Let

$$
q_{k}= \begin{cases}1, & \text { if } k \in M \\ 2, & \text { otherwise }\end{cases}
$$

Let $P_{0}=\left\{\left(x_{k}\right): x_{k}=0\right.$ or 1 , for $k \in M$ and $x_{k}=0$, otherwise\}.

Clearly $P_{0}$ is uncountable.

Consider the class of open balls $B_{1}=\left\{B(z, 1 / 2): z \in P_{0}\right\}$.

Let $C_{1}$ be an open cover of $m_{\mathscr{Z}}^{I}(q)$ containing $B_{1}$.

Since $B_{1}$ is uncountable, so $C_{1}$ cannot be reduced to a countable subcover for $m_{\mathscr{Z}}^{I}(q)$.

Thus $m_{\mathscr{Z}}^{I}(q)$ is not separable.

Theorem 17. Let $G=\sup _{k} q_{k}<\infty$ and I an admissible ideal. Then the following is equivalent.

(a) $\left(x_{k}\right) \in \mathscr{Z}^{I}(q)$;

(b) there exists $\left(y_{k}\right) \in \mathscr{Z}(q)$ such that $x_{k}=y_{k}$, for a.a.k.r.I;

(c) there exists $\left(y_{k}\right) \in \mathscr{Z}(q)$ and $\left(x_{k}\right) \in \mathscr{Z}_{0}^{I}(q)$ such that $x_{k}=y_{k}+z_{k}$ for all $k \in \mathbb{N}$ and $\left\{k \in \mathbb{N}:\left|y_{k}-L\right|^{q_{k}}\right) \geq$ $\epsilon\} \in I$;

(d) there exists a subset $K=\left\{k_{1}<k_{2}<\cdots\right\}$ of $\mathbb{N}$ such that $K \in £(I)$ and $\lim _{n \rightarrow \infty}\left|x_{k_{n}}-L\right|^{q_{k_{n}}}=0$. 
Proof. (a) implies (b).

Let $\left(x_{k}\right) \in \mathscr{Z}^{I}(q)$. Then there exists $L \in \mathbb{C}$ such that

$$
\left\{k \in \mathbb{N}:\left|x_{k}^{\prime}-L\right|^{q_{k}} \geq \epsilon\right\} \in I \text {. }
$$

Let $\left(m_{t}\right)$ be an increasing sequence with $m_{t} \in \mathbb{N}$ such that

$$
\left\{k \leq m_{t}:\left|x_{k}^{\prime}-L\right|^{q_{k}} \geq t^{-1}\right\} \in I .
$$

Define a sequence $\left(y_{k}\right)$ as

$$
y_{k}=x_{k}, \quad \forall k \leq m_{1} .
$$

For $m_{t}<k \leq m_{t+1}, t \in \mathbb{N}$.

$$
y_{k}= \begin{cases}x_{k}, & \text { if }\left|x_{k}^{\prime}-L\right|^{q_{k}}<t^{-1}, \\ L, & \text { otherwise. }\end{cases}
$$

Then $\left(y_{k}\right) \in \mathscr{Z}(q)$ and form the following inclusion:

$$
\left\{k \leq m_{t}: x_{k} \neq y_{k}\right\} \subseteq\left\{k \leq m_{t}:\left|x_{k}^{\prime}-L\right|^{q_{k}} \geq \epsilon\right\} \in I,
$$

we get $x_{k}=y_{k}$, for a.a.k.r.I.

(b) implies (c).

For $\left(x_{k}\right) \in \mathscr{Z}^{I}(q)$. Then there exists $\left(y_{k}\right) \in \mathscr{Z}(q)$ such that $x_{k}=y_{k}$, for a.a.k.r.I.

Let $K=\left\{k \in \mathbb{N}: x_{k} \neq y_{k}\right\}$, then $k \in I$.

Define a sequence $\left(z_{k}\right)$ as

$$
z_{k}= \begin{cases}x_{k}-y_{k}, & \text { if } k \in K, \\ 0, & \text { otherwise. }\end{cases}
$$

Then $z_{k} \in \mathscr{Z}_{0}^{I}(q)$ and $y_{k} \in \mathscr{Z}(q)$.

(c) implies (d).

Suppose (c) holds.

Let $\epsilon>0$ be given.

Let $P_{1}=\left\{k \in \mathbb{N}:\left|z_{k}^{/}\right|^{q_{k}} \geq \epsilon\right\} \in I$ and

$$
K=P_{1}^{c}=\left\{k_{1}<k_{2}<k_{3}<\cdots\right\} \in \mathfrak{E}(I) .
$$

Then we have $\lim _{n \rightarrow \infty}\left|x_{k_{n}}^{l}-L\right|^{q_{k_{n}}}=0$.

(d) implies (a).

Let $K=\left\{k_{1}<k_{2}<k_{3}<\cdots\right\} \in \mathfrak{E}(I)$ and $\lim _{n \rightarrow \infty} \mid x_{k_{n}}^{l}$ $\left.L\right|^{q_{k_{n}}}=0$.

Then for any $\epsilon>0$, and Lemma 11, we have

$$
\left\{k \in \mathbb{N}:\left|x_{k}^{l}-L\right|^{q_{k}} \geq \epsilon\right\} \subseteq K^{\mathcal{c}} \cup\left\{k \in K:\left|x_{k}^{l}-L\right|^{q_{k}} \geq \epsilon\right\} .
$$

Thus $\left(x_{k}\right) \in \mathscr{Z}^{I}(q)$.

Theorem 18. Let $h=\inf _{k} q_{k}$ and $G=\sup _{k} q_{k}$. Then the following results are equivalent.
(a) $G<\infty$ and $h>0$.
(b) $\mathscr{Z}_{0}^{I}(q)=\mathscr{Z}_{0}^{I}$.

Proof. Suppose that $G<\infty$ and $h>0$, then the inequalities $\min \left\{1, s^{h}\right\} \leq s^{q_{k}} \leq \max \left\{1, s^{G}\right\}$ hold for any $s>0$ and for all $k \in \mathbb{N}$.

Therefore the equivalence of (a) and (b) is obvious.

Theorem 19. Let $\left(q_{k}\right)$ and $\left(r_{k}\right)$ be two sequences of positive real numbers. Then $m_{\mathscr{I}_{0}}^{I}(q) \supseteq m_{\mathscr{I}_{0}}^{I}(r)$ if and only if $\lim _{k \in K} \inf \left(q_{k} / r_{k}\right)>0$, where $K^{c} \subseteq \mathbb{N}$ such that $K \in I$.

Proof. Let $\lim _{k \in K} \inf \left(q_{k} / r_{k}\right)>0$ and $\left(x_{k}\right) \in m_{\mathscr{E}_{0}}^{I}(r)$. Then there exists $\beta>0$ such that $q_{k}>\beta r_{k}$, for all sufficiently large $k \in K$.

Since $\left(x_{k}\right) \in m_{\mathscr{I}_{0}}^{I}(r)$ for a given $\epsilon>0$, we have

$$
B_{0}=\left\{k \in \mathbb{N}:\left|x_{k}\right|^{r_{k}} \geq \epsilon\right\} \in I .
$$

Let $G_{0}=K^{c} \cup B_{0}$ Then $G_{0} \in I$.

Then for all sufficiently large $k \in G_{0}$,

$$
\left\{k \in \mathbb{N}:\left|x_{k}\right|^{q_{k}} \geq \epsilon\right\} \subseteq\left\{k \in \mathbb{N}:\left|x_{k}\right|^{\beta r_{k}} \geq \epsilon\right\} \in I .
$$

Therefore $\left(x_{k}\right) \in m_{\mathscr{Z}_{0}}^{I}(q)$.

The converse part of the result follows obviously.

Theorem 20. Let $\left(q_{k}\right)$ and $\left(r_{k}\right)$ be two sequences of positive real numbers. Then $m_{\mathscr{E}_{0}}^{I}(r) \supseteq m_{\mathscr{E}_{0}}^{I}(q)$ if and only if $\lim _{k \in K}$ $\inf \left(r_{k} / q_{k}\right)>0$, where $K^{c} \subseteq \mathbb{N}$ such that $K \in I$.

Proof. The proof follows similarly as the proof of Theorem 19.

Theorem 21. Let $\left(q_{k}\right)$ and $\left(r_{k}\right)$ be two sequences of positive real numbers. Then $m_{0}^{I}(r)=m_{0}^{I}(q)$ if and only if $\lim _{k \in K} \inf \left(q_{k} / r_{k}\right)>0$, and $\lim _{k \in K} \inf \left(r_{k} / q_{k}\right)>0$, where $K \subseteq \mathbb{N}$ such that $K^{c} \in I$.

Proof. By combining Theorems 19 and 20, we get the required result.

\section{Acknowledgment}

The authors would like to record their gratitude to the reviewer for his careful reading and making some useful corrections which improved the presentation of this paper.

\section{References}

[1] I. J. Maddox, "Some properties of paranormed sequence spaces," Journal of the London Mathematical Society, vol. 1, pp. 316-322, 1969.

[2] C. G. Lascarides, "A study of certain sequence spaces of Maddox and a generalization of a theorem of Iyer," Pacific Journal of Mathematics, vol. 38, pp. 487-500, 1971.

[3] C. G. Lascarides, "On the equivalence of certain sets of sequences," Indian Journal of Mathematics, vol. 25, no. 1, pp. 41-52, 1983.

[4] B. Altay, F. Başar, and M. Mursaleen, "On the Euler sequence spaces which include the spaces $l_{p}$ and $l_{\infty}$. I," Information Sciences, vol. 176, no. 10, pp. 1450-1462, 2006. 
[5] F. Başar and B. Altay, "On the space of sequences of $p$ bounded variation and related matrix mappings," Ukrainian Mathematical Journal, vol. 55, no. 1, pp. 136-1147, 2003.

[6] E. Malkowsky, "Recent results in the theory of matrix transformations in sequence spaces," Matematichki Vesnik, vol. 49, no. 3-4, pp. 187-196, 1997.

[7] P. N. Ng and P. Y. Lee, "Cesàro sequence spaces of non-absolute type," Commentationes Mathematicae (Prace Matematyczne), vol. 20, no. 2, pp. 429-433, 1978.

[8] C. S. Wang, "On Nörlund sequence spaces," Tamkang Journal of Mathematics, vol. 9, no. 2, pp. 269-274, 1978.

[9] M. Şengönül, “On the Zweier sequence space," Demonstratio Mathematica, vol. 40, no. 1, pp. 181-196, 2007.

[10] H. Fast, "Sur la convergence statistique," Colloquium Mathematicum, vol. 2, pp. 241-244, 1951.

[11] R. C. Buck, "Generalized asymptotic density," American Journal of Mathematics, vol. 75, pp. 335-346, 1953.

[12] I. J. Schoenberg, "The integrability of certain functions and related summability methods," The American Mathematical Monthly, vol. 66, pp. 361-375, 1959.

[13] J. S. Connor, "The statistical and strong $P$-Cesàro convergence of sequences," Analysis, vol. 8, no. 1-2, pp. 47-63, 1988.

[14] J. S. Connor, "On strong matrix summability with respect to a modulus and statistical convergence," Canadian Mathematical Bulletin, vol. 32, no. 2, pp. 194-198, 1989.

[15] J. Connor, J. Fridy, and J. Kline, "Statistically pre-Cauchy sequences," Analysis, vol. 14, no. 4, pp. 311-317, 1994.

[16] P. Kostyrko, T. Šalát, and W. Wilczyński, “I-convergence,” Real Analysis Exchange, vol. 26, no. 2, pp. 669-686, 2000.

[17] T. Šalát, B. C. Tripathy, and M. Ziman, "On some properties of $I$ convergence," Tatra Mountains Mathematical Publications, vol. 28, no. 2, pp. 279-286, 2004.

[18] T. Šalát, B. C. Tripathy, and M. Ziman, "On I-convergence field," Italian Journal of Pure and Applied Mathematics, no. 17, pp. 45-54, 2005.

[19] K. Demirci, "I-limit superior and limit inferior," Mathematical Communications, vol. 6, no. 2, pp. 165-172, 2001.

[20] B. C. Tripathy and B. Hazarika, "Paranorm I-Convergent sequence spaces," Mathematica Slovaca, vol. 59, no. 4, pp. 485-494, 2009.

[21] B. C. Tripathy and B. Hazarika, "Some I-Convergent sequence spaces defined by Orlicz functions," Acta Mathematicae Applicatae Sinica, vol. 27, no. 1, pp. 149-154, 2011.

[22] V. A. Khan and K. Ebadullah, "On some I-Convergent sequence spaces defined by a modulus function," Theory and Applications of Mathematics and Computer Science, vol. 1, no. 2, pp. 22-30, 2011.

[23] V. A. Khan, K. Ebadullah, and A. Ahmad, "I-pre-Cauchy sequences and Orlicz functions," Journal of Mathematical Analysis, vol. 3, no. 1, pp. 21-26, 2012.

[24] V. A. Khan and K. Ebadullah, "I-Convergent difference sequence spaces defined by a sequence of moduli," Journal of Mathematical and Computational Science, vol. 2, no. 2, pp. 265-273, 2012.

[25] V. A. Khan and K. Ebadullah, "Zweier I-Convergent sequence spaces," submitted to Acta Mathematica Scientia. 


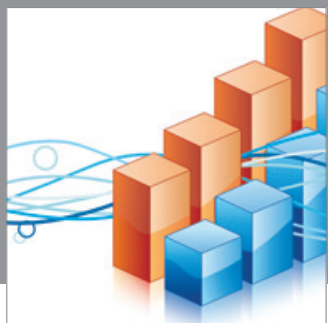

Advances in

Operations Research

mansans

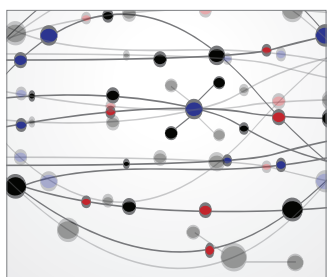

The Scientific World Journal
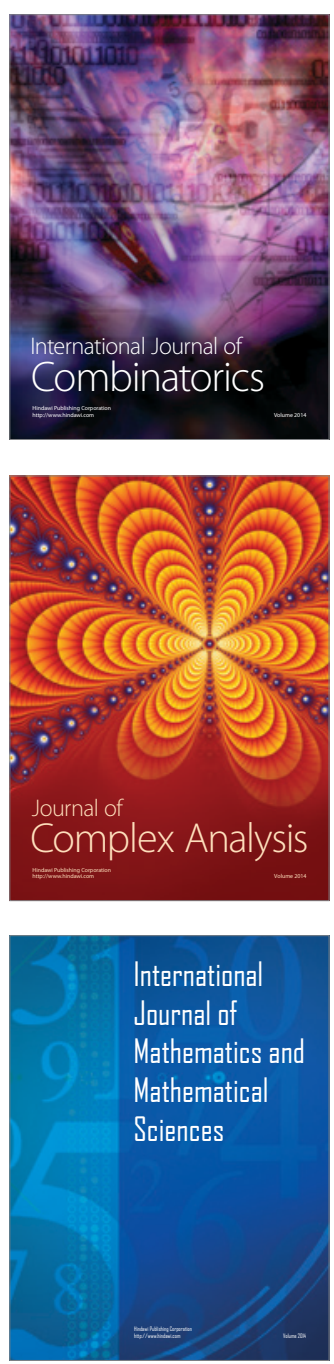
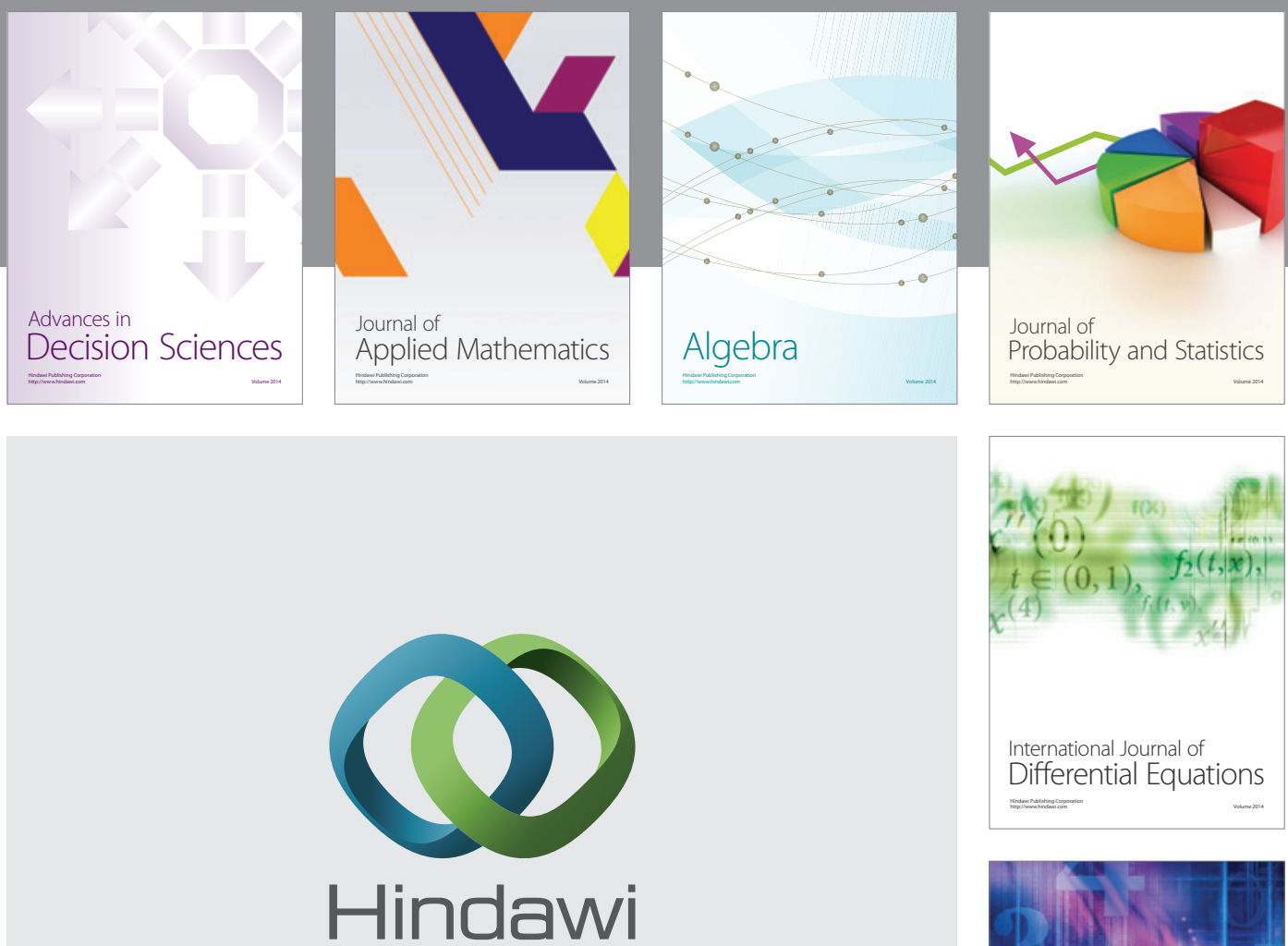

Submit your manuscripts at http://www.hindawi.com
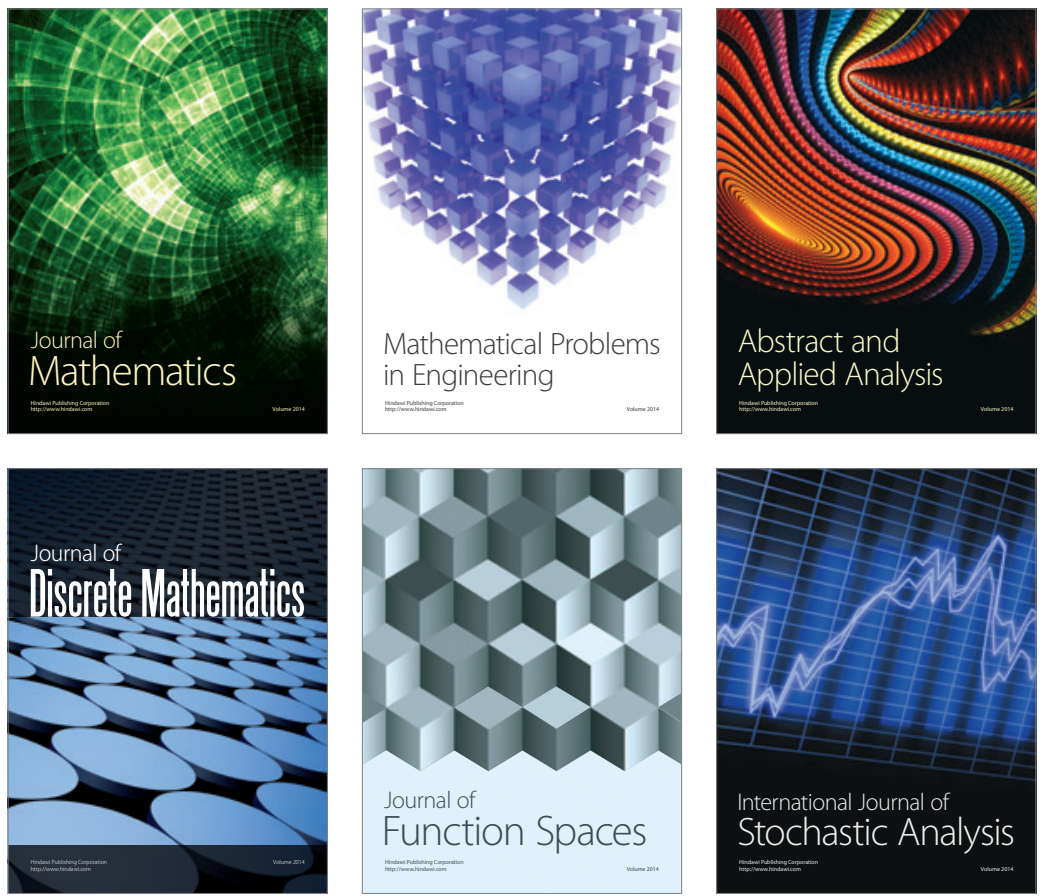

Journal of

Function Spaces

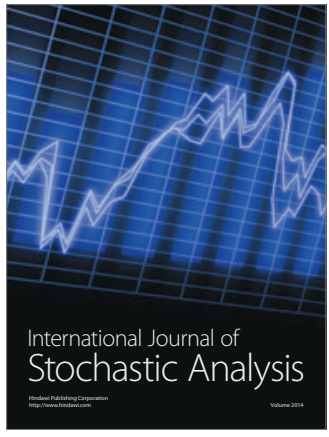

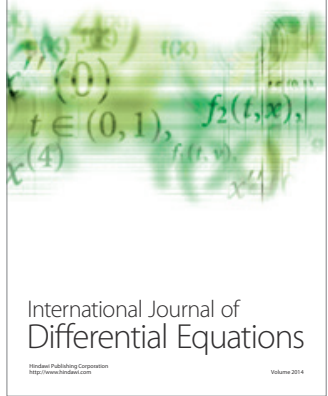
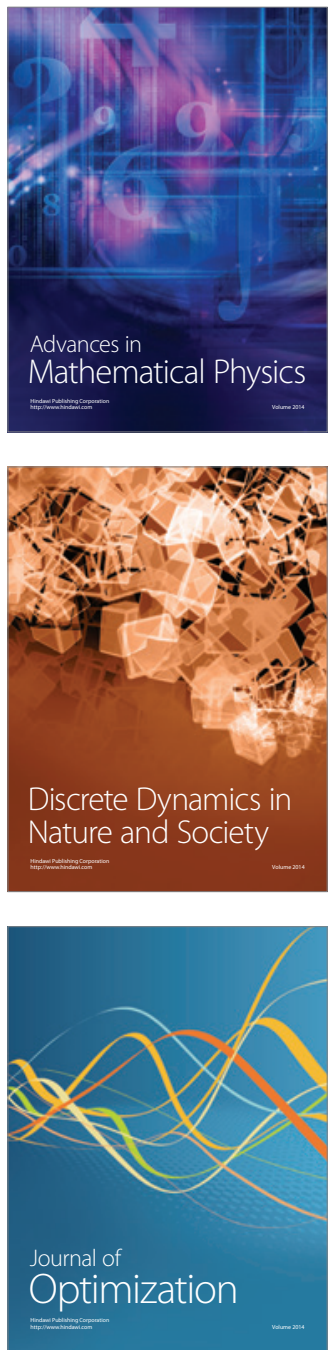